\title{
Efecto moderador de la institucionalización en las actividades de la vida diaria y bienestar en el envejecimiento
}

\author{
Moderator effect of living in nursing homes on well-being and activities of daily life in the elderly
}

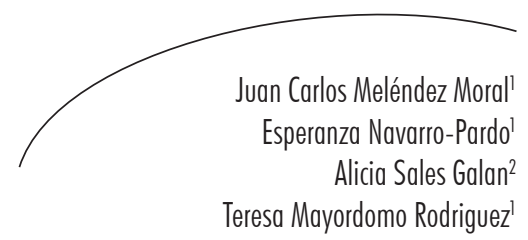

\section{Resumen}

Objetivo: El envejecimiento con éxito es un concepto multidimensional que se puede evaluar a partir de componentes objetivos y subjetivos como el funcionamiento físico, psicológico y social. El objetivo de este trabajo es comparar el funcionamiento en variables relacionadas con el envejecimiento exitoso en ancianos institucionalizados y no institucionalizados. Método: Se realizó un diseño de dos grupos independientes, institucionalizados $(\mathrm{N}=242)$ y no institucionalizados $(\mathrm{N}=607)$, todos de más de 65 años, evaluándose sus actividades de la vida diaria (AVD) y el bienestar psicológico. Resultados: El Índice de Barthel mostró diferencias significativas, siendo inferior la media de los institucionalizados que la de los no institucionalizados. En relación al bienestar, el MANOVA mostró diferencias entre grupos para todas las escalas. En los posteriores ANOVA's la variable crecimiento personal mostró las mayores diferencias y porcentaje de varianza explicada. Se obtuvieron correlaciones significativas de la edad con el Índice de Barthel, y con las siguientes escalas de bienestar psicológico de Ryff: autoaceptación, relaciones positivas con otros, dominio del ambiente y propósito en la vida. Conclusiones: Los institucionalizados muestran puntuaciones significativamente inferiores en AVD y en bienestar psicológico, actuando como variable moderadora, que puede empeorar su situación. La edad afecta de forma más clara al bienestar de los institucionalizados, reafirmándose la necesidad de generar estrategias que mejoren la adaptación de las personas.

Abstract

Objectives: Successful aging is a multidimensional concept that can be evaluated from objective and subjective components, such as physical, psychological and social functioning. This study aimed to compare the performance on variables related to
Palabras clave:

Envejecimiento. Calidad de vida. Actividades de la vida diaria. Bienestar.

\footnotetext{
Departamento de Psicología Evolutiva y de la Educación, Facultad de Psicología. Universidad de Valencia. Valencia, España.

2 Departamento de Metodología de las Ciencias del Comportamiento, Facultad de Psicología. Universidad de Valencia, España. 
successful aging in elderly institutionalized and non-institutionalized. Methods: We conducted a design of two independent groups, institutionalized $(\mathrm{N}=242)$ and noninstitutionalized $(\mathrm{N}=607)$, all aged over 65 years, evaluating their activities of daily living (ADL) and psychological well being. Results: The Barthel index showed significant differences, being the mean of the institutionalized group lower than the mean of the non- institutionalized group. Regarding well-being, MANOVA showed differences between groups for all the scales. In subsequent ANOVA's, the variable personal growth showed the greatest differences and the percentage of explained variance. There were significant correlations between age and the Barthel Index, and the following scales Ryff's psychological well-being: self-acceptance, positive relations with others, environmental mastery and purpose in life. Conclusions: The institutionalized group has significantly lower scores in ADL and psychological well-being, and therefore living in nursing homes acts as a moderating variable that can worsen the situation. Additionally, age clearly affects the well-being of the institutionalized group, strengthening the need for generating strategies that improve the adaptation of the elderly.
Key words: Aging. Quality of life. Activities of daily life. Well-being.

\section{INTRODUCCIÓN}

El concepto de envejecimiento satisfactorio ha evolucionado desde un modelo centrado exclusivamente en aspectos físicos hacia otro mucho más integrador, en el que la calidad de vida se convierte en una de las prioridades del desarrollo de las personas mayores y que se define incluyendo la baja probabilidad de enfermedad y de discapacidad asociada, el alto funcionamiento cognitivo y funcionalidad física y el compromiso activo con la vida. ${ }^{1,2}$ De este modo, nos encontramos ante un concepto multidimensional que depende de factores personales, del entorno y de su interacción, y que integra componentes tanto objetivos como subjetivos.

Respecto a estos componentes, aquellos de corte más objetivo, hacen referencia a características claramente medibles, lo que proporciona información comparable mediante escalas de rendimiento, funcionalidad $\mathrm{O}$ dependencia, y nos proporcionan unos criterios más exactos de intervención impidiendo a la vez que consideremos que todo está bien si la persona así lo considera. ${ }^{3}$ Entre éstos se encuentran aspectos como las actividades de la vida diaria (AVD) o el estado cognitivo de la persona.

En este sentido, la evaluación de las AVD proporciona información acerca de trastornos que no son atribuibles a una patología específica, pero que generan un aumento en el riesgo de dependencia por pérdida de la autonomía y la independencia elementales y que permiten vivir sin precisar ayuda continua de otros. Hemos de tener en cuenta que en los próximos años se producirá un importante envejecimiento demográfico en España y con ello habrá un mayor nivel de discapacidad y dependencia. ${ }^{4}$

El segundo tipo de criterios, de corte más subjetivo, implican la opinión y los valores de la persona y hacen referencia a aspectos emocionales o evaluativos que nacen de la apreciación personal, lo que influye positivamente en las intervenciones objetivas propuestas. Dentro de este tipo de criterios el estudio del bienestar ha supuesto el elemento central de la investigación científica. ${ }^{5-9}$

En lo referente al bienestar, la literatura científica psicológica ha mantenido dos líneas de trabajo paralelas centradas en lo que ha venido definiéndose como bienestar psicológico y bienestar subjetivo. El bienestar psicológico es descrito $^{10}$ como un esfuerzo por perfeccionarse y conseguir la realización del propio potencial. Así, su desarrollo está altamente relacionado con tener un propósito en la vida, con que la vida adquiera significado para uno mismo, con los desafíos y con un cierto esfuerzo por superarlos y conseguir metas valiosas. El bienestar subjetivo es cercano a conceptos de larga tradición en la psicología y, actualmente, engloba términos como 
felicidad, moral o satisfacción vital, quedando definido $^{11}$ como un constructo compuesto por un componente emocional o afectivo y otro de carácter cognitivo, estando el primero relacionado con los sentimientos de placer y displacer que experimenta la persona (felicidad) y el segundo referido al juicio que merece a la persona su trayectoria evolutiva (satisfacción).

Parece ser que el bienestar subjetivo y el psicológico están relacionados, ${ }^{7}$ y aunque ambos poseen dimensiones que no comparten con el otro, existe un cierto solapamiento entre ellos en las dimensiones de autoaceptación y dominio del ambiente, a la par que el bienestar psicológico mantiene dimensiones que son exclusivas, como propósito en la vida y crecimiento personal.

De este modo, nos planteamos como objetivo de este trabajo comparar el funcionamiento en variables relacionadas con el envejecimiento exitoso en ancianos institucionalizados y no institucionalizados, planteándose como primera hipótesis que deben existir diferencias significativas en el nivel de AVD entre ancianos institucionalizados y no institucionalizados. Posteriormente, se analizarán las diferentes dimensiones del bienestar para comprobar si existen diferencias entre ambos grupos, planteándose como segunda hipótesis que los niveles de las dimensiones de bienestar de los sujetos institucionalizados deberán ser inferiores. En este sentido, conocer si existen diferencias en ambas variables podría ser determinante para desarrollar futuras intervenciones dirigidas a la mejora, tanto física como psicológica del estado de los ancianos, lo cual supondría una reducción o mantenimiento del nivel de dependencia, un aumento de su bienestar y, por tanto, una mejora de la salud de este grupo de edad.

\section{MÉTODOS}

\section{Participantes}

La muestra está compuesta por dos grupos independientes, institucionalizados $(\mathrm{N}=242)$ y no institucionalizados ( $\mathrm{N}=607)$, estableciéndose como criterio de inclusión que tuvieran 65 ó más años y que residiesen en la provincia de Valencia (España). La diferencia en el número de sujetos de las dos submuestras viene determinada por la diferente representación de las mismas en el contexto español. De este modo, y para el desarrollo de la investigación se planteó un diseño de dos grupos con encuesta, debido a que las características de la muestra sólo pueden ser observadas tal y como se presentan; de este modo, no se podía asignar aleatoriamente a los sujetos a los valores de la variable independiente, que es intrínseca al sujeto y no puede ser modificada; así, la asignación a los grupos se produce por poseer un determinado valor en dicha variable, que en el caso de este trabajo era estar o no institucionalizados.

En relación al muestreo, en el caso de la población no institucionalizada, se obtuvo la participación voluntaria de sujetos que asistían a centros y asociaciones de personas mayores. En el caso de las personas institucionalizadas, se contó con la colaboración de cuatro residencias. En este caso y debido a las dificultades cognitivas de parte de los participantes, evaluadas mediante el MMSE, los instrumentos de autocompletados sólo pudieron ser contestados por el 38,2\%, si bien para las actividades de la vida diaria se dispuso del total de la muestra.

Se obtuvo el consentimiento informado de los participantes en el estudio siguiendo las recomendaciones de la declaración de Helsinki.

\section{Medidas}

En relación a los instrumentos utilizados, además de una plantilla con diferentes elementos sociodemográficos, se aplicó la versión adaptada al español del Índice de Barthel, ${ }^{12}$ y las Escalas de Bienestar Psicológico de Ryff. ${ }^{5}$

El Índice de Barthel es una medida de la discapacidad física con demostrada validez y fiabilidad, fácil de aplicar y de interpretar y cuyo uso rutinario es recomendable, siendo útil para 
valorar la discapacidad funcional en las AVD, sobre todo en población anciana. Este índice está constituido por 10 ítems que valoran la capacidad de realizar ciertas actividades (alimentación, baño, vestido, aseo personal, continencia urinaria, continencia fecal, uso del retrete, traslados, deambulación, y uso de escaleras), y se puntúa de forma cuantitativa en una escala de 0 (completamente dependiente) a 100 (completamente independiente). La puntuación cuantitativa permite una categorización de los participantes en 4 grupos, de carácter ordinal según su nivel de capacidad funcional: severa (0-45), grave (4659), moderada (60-80) y ligera (81-100).

Para la valoración del bienestar psicológico se aplicaron las Escalas de Bienestar Psicológico de Ryff que en muestras de personas mayores ha mostrado resultados psicométricos relativamente satisfactorios. ${ }^{13}$ Para el desarrollo de este trabajo se aplicó la versión validada en población española y reducida a 29 ítems ${ }^{14}$ debido a las características de la propia muestra y que parte de la escala original de 84 ítems. Esta versión reducida tiene un número variable de ítems por factor y al igual que en la escala original, el formato de respuesta utilizado tiene puntuaciones comprendidas entre 1 (totalmente en desacuerdo) y 6 (totalmente de acuerdo). Las escalas que la componen son autonomía, dominio del ambiente, autoaceptación, relaciones positivas con otros, crecimiento personal y propósito en la vida.

\section{Análisis}

Se han calculado estadísticos descriptivos, en concreto, medias, desviaciones típicas y porcentajes de las diferentes variables estudiadas. Por lo que se refiere a la comparación entre grupos, para el análisis de las AVD tomadas de forma cuantitativa, se aplicó la t de Student para la comparación de dos medias en muestras independientes, analizando la homogeneidad de varianzas mediante la prueba de Levene. Para el análisis de las medidas de bienestar y partiendo de la alta correlación entre las escalas, se optó por la aplicación de un MANOVA para estudiar de forma multivariada si existían diferencias significativas entre las medias, dado la existencia de más de una variable dependiente para los dos grupos de estudio; como contrastes multivariados se aplicaron la lambda de Wilks y la traza de Pillai para evaluar si había diferencias entre las medias de la combinación lineal de variables. Caso de observar diferencias significativas, se opta por evaluar mediante ANOVAs de continuación la significación para cada una de las variables por separado, comparándose por tanto la media de los grupos en la variable, e incluyendo un cálculo del tamaño del efecto mediante eta-cuadrado $\left(\eta^{2}\right)$ como estimador de la proporción de varianza explicada. En relación a la comparación de ambos grupos en las correlaciones de Pearson obtenidas entre la edad y las variables analizadas, se aplicó la formula de comparación de coeficientes para muestras independientes ${ }^{15}$. A partir de esta fórmula se obtienen puntuaciones $\mathrm{Z}$, las cuales nos indican el valor del estadístico de contraste que determina la existencia o no de diferencias significativas entre correlaciones, de manera que cuando el valor supere $\pm 1,96$ tendrá una significación de $\mathrm{p}<, 05$ y cuando supere $\pm 2,54$ tendrá una significación de $\mathrm{p}<, 01$.

\section{RESULTADOS}

En primer lugar y a nivel descriptivo se incluye en la tabla 1 la información básica de los grupos de estudio en sus principales características sociodemográficas. 
Tabla 1 - Principales características sociodemográficas de los grupos. Valencia (España), 2011.

\begin{tabular}{lcc}
\hline & No institucionalizados & Institucionalizados \\
\hline $\mathrm{N}$ & 607 & 242 \\
Edad media & $76,8( \pm 7,4)$ & $81,99( \pm 7,5)$ \\
Género & & \\
Hombres & $39,5 \%$ & $22,15 \%$ \\
Mujeres & $60,5 \%$ & $77,85 \%$ \\
Estado civil & & \\
Casado & $56,6 \%$ & $19,7 \%$ \\
Viudos & $32,6 \%$ & $63,4 \%$ \\
Otros & $10,7 \%$ & $16,9 \%$ \\
Nivel de estudios & & \\
Sin estudios & $18,3 \%$ & $32,9 \%$ \\
Primarios & $55,4 \%$ & $60,5 \%$ \\
Secundarios & $16,6 \%$ & $4,9 \%$ \\
Universitarios & $9,7 \%$ & $1,6 \%$ \\
\hline
\end{tabular}

Por lo que se refiere a la primera de las hipótesis planteada, la comparación de los grupos en las AVD, la prueba $t$ de muestras independientes aplicada a la puntuación cuantitativa obtenida mediante el Índice de Barthel, mostró diferencias significativas entre los grupos $(\mathrm{p}<, 001)$, siendo inferior la media del grupo de institucionalizados de 47,02 (DE 32,67) que la de los no institucionalizados, de 91,44 (DE 15,7). De forma descriptiva y por lo que se refiere a la información cuantitativa proporcionada por la escala, se presenta la tabla 1 en la que se compara el porcentaje de sujetos en cada una de las categorías.

Por lo que respecta a las escalas de bienestar, al realizar el MANOVA, la lambda de Wilks y la traza de Pillai obtuvieron un resultados significativo $\left(\mathrm{F}_{6,693}=18,136 ; \mathrm{p}<, 000 ; \square^{2}=\right.$ ,136), señalando la existencia de diferencias entre ambos grupos en el conjunto de variables dependientes. En la tabla 2, se muestran los principales resultados de los ANOVA's, que también mostraron la existencia de diferencias significativas para cada una de las escalas.

Tal y como se observa en la tabla 2, es la variable crecimiento personal en la que los grupos difieren más en las medias, tal y como puede observarse a partir de la proporción de varianza explicada, mientras que autoaceptación, dominio del ambiente, relaciones positivas con otros y propósito en la vida obtienen proporciones de varianza explicada menores y más similares. Además, las medias de los no institucionalizados son superiores en todos los casos. 
Tabla 2 - Principales resultados del ANOVA de las escalas de bienestar. Valencia (España), 2011

\begin{tabular}{lccccccc}
\hline \multicolumn{1}{c}{ Escala } & Grupo & N & Media & DE & F & p & $\eta^{2}$ \\
\hline Autoaceptación & Institucionalizado & 93 & 3,96 & 0,95 & 44,807 &, 000 & 0,060 \\
& No institucionalizado & 607 & 4,66 & 0,92 & & & \\
Relaciones Positivas & Institucionalizado & 93 & 3,66 & 0,86 & 37,780 &, 000 & 0,051 \\
& No institucionalizado & 607 & 4,31 & 0,96 & & & \\
Autonomía & Institucionalizado & 93 & 3,94 & 0,77 & 18,766 &, 000 & 0,026 \\
& No institucionalizado & 607 & 4,36 & 0,89 & & & \\
Domino Ambiente & Institucionalizado & 93 & 3,67 & 0,81 & 39,787 & \multirow{2}{*}{000} & 0,054 \\
& No institucionalizado & 607 & 4,28 & 0,87 & & & \\
Crecimiento Personal & Institucionalizado & 93 & 3,73 & 0,89 & 96,101 &, 000 & 0,121 \\
& No institucionalizado & 607 & 4,67 & 0,85 & & & \\
Propósito Vida & Institucionalizado & 93 & 3,77 & 0,89 & 31,667 & \multirow{2}{*}{000} & 0,043 \\
& No institucionalizado & 607 & 4,37 & 0,96 & & & \\
\hline
\end{tabular}

$\mathrm{DE}=$ desviación estándar; $\eta^{2}=$ eta-cuadrado

Por lo que respecta a las correlaciones entre la edad y las variables dependientes estudiadas, la comparación entre los grupos de institucionalizados y no institucionalizados mostró diferencias significativas para las siguientes escalas de bienestar: autoaceptación, relaciones positivas con otros, dominio del ambiente y propósito en la vida. En la tabla 3 se muestra el estadístico de contraste en puntuación $Z$ así como la significación de la comparación de los coeficientes de correlación obtenidos entre la edad y las variables dependientes en ambos grupos.

Tal y como se observa en la tabla 3 , al comparar los coeficientes de las correlaciones obtenidas entre la edad y las diferentes variables, han sido elementos del bienestar los que han mostrado diferencias significativas entre los dos grupos, mientras que la valoración de las AVD no ha mostrado diferencias.

Tabla 3 - Significación del estadístico de contraste obtenido tras la comparación de los coeficientes de correlación. Valencia (España), 2011.

\begin{tabular}{lcc}
\hline \multicolumn{1}{c}{ Medidas } & $\mathrm{Z}$ & $\mathrm{p}$ \\
\hline Índice de Barthel & $-0,740$ & --- \\
Autoaceptación & $-3,828$ & $\mathrm{p}<, 01$ \\
Relaciones Positivas & $-2,561$ & $\mathrm{p}<, 01$ \\
Autonomía & $-0,045$ & --- \\
Domino Ambiente & $-2,781$ & $\mathrm{p}<, 01$ \\
Crecimiento Personal & $-1,457$ & --- \\
Propósito Vida & $-5,036$ & $\mathrm{p}<, 01$ \\
\hline
\end{tabular}




\section{DISCUSIÓN}

La aportación más importante de este trabajo es la demostración de la existencia de diferencias entre dos grupos de personas mayores (institucionalizadas y no institucionalizadas) respecto a variables relacionadas con el envejecimiento con éxito y la calidad de vida como son las AVD y el bienestar, observándose además una significativa disminución de las puntuaciones de aquellas personas que se encuentran institucionalizadas.

En otro orden de cosas, destacan las diferencias en los datos sociodemográficos de los grupos que componen la muestra, observándose que el grupo de personas institucionalizadas posee una edad superior, con un porcentaje más alto de mujeres, siendo la mayoría personas viudas y con más problemas de carácter cognitivo. Todo esto refuerza los planteamientos en relación a la utilización de cuidados formales, de manera que el aumento de la edad genera una reducción de la composición y el tamaño de la red, así, a medida que la edad aumenta se reduce el número de potenciales cuidadores, además, las mujeres tienen más probabilidad de recibir cuidado formal ya que viven más que los hombres y con más enfermedades crónicas discapacitantes, de este modo se observa una mayor prevalencia del modelo de cuidado complementario que aparece cuando las tareas demandadas exceden las capacidades. ${ }^{16}$

Reafirmando esta última idea, hemos podido comprobar a través del análisis de las AVD, cómo el grupo de personas institucionalizadas mantiene una media significativamente inferior, lo cual implica, además de la propia institucionalización, una incidencia mayor de enfermedades agudas y de utilización de servicios sanitarios. Así, parece clave el desarrollo de una mayor autonomía ya que, aunque el envejecimiento sitúa a las personas en un escenario caracterizado por cambios que suponen una situación desventajosa, las posibilidades de adaptación y ajuste pueden conducir hacia un envejecimiento más exitoso. En este sentido, la aplicación de estrategias de tipo acomodativo como forma de adaptación a las nuevas situaciones logra generar un ajuste de las preferencias y de las metas que, aun no siendo intencional, hace que el sujeto se adapte adecuadamente a la nueva situación, paliando la percepción de las restricciones situacionales o unos recursos personales insuficientes para conseguir metas antes valoradas. ${ }^{17}$

En relación a las diferencias existentes entre los grupos respecto al bienestar, hemos podido observar diferencias en todas las escalas, destacando la variable crecimiento personal que, al igual que en otros trabajos, ${ }^{18,19}$ es definida como una de las bases determinantes del bienestar psicológico junto a la variable propósito en la vida. Así, y partiendo de la idea de que el envejecimiento es un suceso vital que puede modificar el bienestar, la institucionalización parece disminuir en mayor medida éste, debido posiblemente a la pérdida de control sobre las situaciones de su vida y de habilidad para manejarlas. ${ }^{20}$ Se debe tener en cuenta que la dimensión psicológica está altamente vinculada con el dominio de la salud, tanto en aspectos objetivos como subjetivos, pero también existe evidencia del efecto protector que el bienestar puede generar sobre la salud, por lo que parece necesario apoyar capacidades personales y dar reconocimiento como formas que favorezcan el afrontamiento.

Por lo que respecta a los resultados de las correlaciones, un primer dato a comentar es que no se han observado diferencias entre los grupos para los coeficientes obtenidos entre la edad y las AVD. Debemos tener en cuenta que en ambos grupos existe una misma tendencia, según la cual a más edad mayor nivel de dependencia, lo cual reafirma la idea de generar estrategias que mejoren la adaptación de las personas al proceso del envejecimiento ya que la edad es una de los principales predictores de la capacidad funcional.

Por otra parte y en relación al bienestar psicológico, la dimensión de propósito en la vida ha sido la que mayores diferencias ha mostrado, lo cual es coherente con la dificultad para establecer 
nuevas metas que probablemente se produce tras la institucionalización; además, en los institucionalizados se ha observado una mayor dificultad para el desarrollo y mantenimiento de relaciones positivas, dominio ambiente y autoaceptación, dimensiones del bienestar que otros trabajos ${ }^{18,19,21}$ realizados exclusivamente con población mayor no institucionalizada, han señalado como elementos con una alta estabilidad incluso en edades avanzadas.

De todo lo planteado hasta aquí, podríamos concluir que la institucionalización es una variable moderadora que puede empeorar la situación de los ancianos. Pero este análisis, demasiado simplista, podría ser causa de interpretaciones erróneas si no se tienen en cuenta otras variables.

De este modo, si bien las personas con peor estado de salud física o cognitiva tienen más probabilidades de ser institucionalizadas, y ello influiría en peores resultados tanto en AVD como en bienestar, numerosos estudios señalan también como causas de la institucionalización la pérdida del cónyuge, la no existencia de hijos o de familiares con disponibilidad para el cuidado, en suma, factores relacionados con la falta de apoyo social, aspecto éste que destaca como fundamental para enfrentarse durante el envejecimiento a situaciones estresantes que requieren adaptación, mostrando además efectos amortiguadores frente al inicio de la discapacidad, frenando su progresión e incluso incrementando el grado de recuperación. ${ }^{22}$

\section{CONCLUSIONES}

Tal y como se ha podido comprobar a partir de los resultados de este trabajo, la institucionalización de los ancianos tiene claras repercusiones de carácter negativo tanto sobre la calidad de vida y realización de actividades básicas de la vida diaria como sobre las diferentes dimensiones del bienestar psicológico, constituyendo por tanto la institucionalización una importante variable moderadora a tener en cuenta en la optimización del envejecimiento.

Además, y teniendo en cuenta las modificaciones demográficas en las pirámides de población, parece necesario planificar a medio y largo plazo las necesidades y cuidados dirigidos a la tercera edad, tanto por cuestiones sociales como económicas, ya que tanto el gasto sanitario como el social ofrecen unas perspectivas de incremento constante para este grupo poblacional.

Todo ello parece incidir en la necesidad de un replanteamiento de la atención a las personas mayores, diversificando los recursos públicos disponibles y no restringiendo la mayor parte de las acciones a instituciones cerradas que conllevan, entre otros efectos negativos, la disminución de actividad física, la sensación de falta de utilidad y de pérdida de control sobre sí mismo y su entorno. Por ello, este tipo de centros, en muchas ocasiones, no constituye el recurso más indicado para personas mayores independientes $\mathrm{o}$ semiindependientes que sólo necesitan ayudas parciales o temporales y para los que la institucionalización resulta contraproducente.

Por todo ello debería ser un objetivo prioritario que las personas mayores permanezcan en sus entornos habituales de convivencia e integrados en su comunidad, desarrollando otro tipo de intervenciones que pueden aumentar su bienestar y calidad de vida, tanto a nivel físico como psicológico, de modo que la institucionalización sólo se produzca cuando constituya la mejor alternativa pero no la de primera elección. ${ }^{16}$

Como recursos que fomentan esta permanencia, señalaremos, por ejemplo, las redes municipales de asistencia a domicilio, las ayudas para adecuación de las viviendas propias, especialmente en cuanto a accesibilidad, los acogimientos familiares, las viviendas asistidas, la teleasistencia gratuita, los centros residenciales, con servicios comunes generales y específicos para mayores, los centros de día públicos, incluso 
los programas de convivencia intergeneracional, etc. Unos servicios alternativos podrían permitir, en algunos casos, permanecer en su propio hogar y contexto a personas que actualmente se encuentran institucionalizadas.

Finalmente, indicar que este estudio presenta una serie de limitaciones que futuros trabajos debieran intentar superar, como son el muestreo no probabilístico y su carácter transversal, lo que dificulta la generalización de los resultados, efectos que podrían minimizarse a partir de una evaluación longitudinal de personas mayores antes y después de ser institucionalizados, añadiendo evidencia empírica a este muestreo de un solo momento temporal. Por otra parte, la muestra institucionalizada, ha sufrido una drástica reducción debido a problemas de carácter cognitivo, lo cual en el caso de los instrumentos autocompletados reduce la potencia de los resultados.

\section{REFERENCIAS}

1. Rowe JW, Kahn RL. Human aging: Usual and successful aging. Science. 1987;237:143-9.

2. Rowe JW, Kahn RL. Successful aging. Gerontologist. 1997;37:433-40.

3. Triadó C, Villar F. Modelos de envejecimiento y percepción de cambios en una muestra de personas mayores. Anu Psicol. 1997;73:43-55.

4. Zunzunegui MV, Béland F. Políticas intersectoriales para abordar el reto del envejecimiento activo. Informe SESPAS 2010. Gac Sanit. 2010;24(Suppl 1):68-73.

5. Ryff CD. Happiness is everything, or is it? Explorations on the meaning of psychological wellbeing. J Pers Soc Psychol. 1989;57:1069-81.

6. Ryff CD, Essex MJ. (). Psychological well-being in adulthood and old age: Descriptive markers and explanatory processes. Annu Rev Gerontol Geriatr. 1991;11:144-71.

7. Keyes C, Shmotkin D, Ryff CD. Optimizing wellbeing: The empirical encounter of two traditions. J Pers Soc Psychol. 2002;82:1007-22.

8. Springer KV, Hauser RM. An assessment of the construct validity of Ryff's Scales of Psychological Well-Being: Method, mode, and measurement effects. Soc Sci Res. 2006;35:1080-102.

9. Springer KV, Hauser RM, Freese J. Bad news indeed for Ryff's six-factor model of well-being. Soc Sci Res. 2006;35:1120-31.

10. Ryff CD, Keyes C. The structure of psychological wellbeing revisited. J Pers Soc Psychol. 1995;69:719-27.
11. Diener E, Lucas RE. Personality and subjective well-being. En: Kahneman D, Diener E, Schwarz $\mathrm{N}$, editors. Well-being: The foundations of hedonic psychology. New York: Russell Sage; 1999. p. 213-27.

12. Baztán JJ, Pérez del Molino J, Alarcón T, San Cristóbal E, Izquierdo G, Manzarbeitia J. Índice de Barthel: Instrumento válido para la valoración funcional de pacientes con enfermedad cerebrovascular. Rev Esp Geriatr Gerontol. 1993;28:32-40.

13. Tomás JM, Meléndez JC, Navarro E. Modelos factoriales confirmatorios de las escalas de Ryff en una muestra de personas mayores. Psicothema. 2008;20:298-304.

14. Díaz D, Rodríguez-Carvajal R, Blanco A, MorenoJiménez B, Gallardo I, Valle C, et al. Adaptación española de las escalas de bienestar psicológico de Ryff. Psicothema. 2006;18:572-77.

15. Amón J. Estadística para psicólogos. Probabilidad estadística inferencia. Madrid: Pirámide; 1988.

16. Rogero-García J. Distribución en España del cuidado formal e informal a las personas de 65 y más años en situación de dependencia. Rev Esp Salud Publica. 2009;83:393-405.

17. Brandtstädter J, Renner G. Tenacious goal pursuit and flexible goal adjustment: Explication and agerelated analysis of assimilative and accommodative strategies of coping. Psychol Aging. 1990;5:58-67.

18. Meléndez JC, Tomás JM, Navarro E. Análisis del bienestar en la vejez según la edad. Rev Esp Geriatr Gerontol. 2008;43:90-5. 
19. Tomás JM, Oliver A, Navarro E, Meléndez JC, Molina C. Comparación del bienestar psicológico de personas mayores dominicanas y españolas. Rev Esp Geriatr Gerontol. 2009;44:180-5.

20. Sáez N, Meléndez JC, Aleixandre M. Variables propiciatorias del incremento de posibilidades: Diferencias entre prejubilados y jubilados. Geriátrika, 1995;11: 371-8.

Recebido: 04/10/2011

Revisado: $17 / 4 / 2012$

Aprovado: 30/5/2012
21. Triadó C, Villar F, Solé C, Osuna MJ. La medida del bienestar en personas mayores: Adaptación de la escala de Ryff. Rev Psicol Gen Apl. 2005;58:347-63.

22. Meléndez JC, Tomás JM, Navarro E. Análisis de las redes sociales en la vejez a través de la entrevista Manhein de apoyo social. Salud Pública México. 2007;49:408-16 